\title{
Utilidad del CMBD para la detección de acontecimientos adversos por medicamentos
}

\author{
L. A. SÁNCHEZ MUÑOZ, J. CASTIELLA HERRERO, F. J. SANJUÁN PORTUGAL, \\ J. NAYA MANCHADO, M. J. ALFARO ALFARO' \\ Servicios de Medicina Interna y ${ }^{\prime}$ Farmacia. Fundación Hospital Calahorra. Calahorra, \\ La Rioja
}

USEFULNESS OF MBDS IN DETECTION OF ADVERSE DRUG EVENTS

\section{RESUMEN}

Objetivo: Analizar la incidencia de los acontecimientos adversos a medicamentos (AAM) notificados en el informe de alta hospitalaria, valorar su evitabilidad y describir los fármacos implicados, las manifestaciones clínicas y los tipos de errores asociados a los AAM prevenibles.

Material y método: Estudio retrospectivo del Conjunto Mínimo Básico de Datos (CMBD) del periodo enero-diciembre de 2005 en todos los servicios asistenciales de un hospital de ámbito comarcal, centrado en los pacientes en cuyo informe de alta se incluían los códigos asociados a efectos adversos por medicamentos según la Clasificación Internacional de Enfermedades (CIE-9-MC)

Resultados: Se detectan AAM en un 4,01\% de las altas codificadas en el período de estudio $(n=160) .45 \%$ de los AAM $(n=72)$ causados por el uso extrahospitalario de fármacos y $55 \%(\mathrm{n}=88)$ durante la hospitalización. El $62,3 \%$ de los AAM eran prevenibles $(\mathrm{n}=109)$. El 38,1\% de los AAM fueron graves, 40,0\% moderados y 21,9\% leves. Los fármacos más frecuentemente implicados en AAM fueron: antiinfecciosos $24,0 \%$, glucocorticoides sistémicos $15,4 \%$, AINEs y otros analgésicos $11,4 \%$, diuréticos $10,3 \%$, digoxina $9,1 \%$, insulina y antidiabéticos orales $5,7 \%$, anticoagulante orales y heparina $5,7 \%$. Las causas que motivaban su aparición fueron la falta de seguimiento $(47,7 \%)$, dosis elevada $(28,5 \%)$, e interacción medicamentosa $(10,1 \%)$

Conclusiones: Un 3,2\% de los ingresos urgentes estaba motivado por AAM. Un 2,2\% de los pacientes hospitalizados presentó AAM. Un $62 \%$ de AAM era prevenible. Un alto grado de AAM prevenibles se centran en un número reducido de fármacos. Es necesario implantar prácticas efectivas de seguridad para reducir los errores de medicación.

PALABRAS CLAVE: Errores de medicación. Acontecimientos adversos a medicamentos. Evitabilidad. Tratamiento farmacológico-efectos adversos. Reacciones adversas a medicamentos.

\section{ABSTRACT}

Objective: To analyze the incidence of adverse drug events (ADE) as noted in hospital discharge reports, as well as their potential avoidability, drugs involved, clinical symptoms and the type of medication errors that led to the preventable ADE.

Material and methods: A retrospective study for the JanuaryDecember 2005 period of time, at a district hospital. ADE were detected in which patients with discharge reports including event codes as defined by the IDC-9-CM system, using the minimum basic data set (MBDS).

Results: ADEs were detected in $4.01 \%$ of all discharge reports in the study period $(n=160) .45 \%$ of ADEs were were detected at the Emergency Department $(n=72)$ and $55 \%(n=88)$ were detected during hospitalization.

$62.3 \%$ of ADEs were considered potentially avoidable $(n=109)$. $38.1 \%$ of ADEs were serious, $40.0 \%$ moderate and $21.9 \%$ mild. Drugs most commonly involved in the ADEs sample studied included: antimicrobials $(24.0 \%)$, systemic corticoids $(15.4 \%)$, NSAIDs $(11.4 \%)$, diuretics $(10.3 \%)$, digoxin $(9.1 \%)$, insulin and oral hypoglycaemic agents $(5.7 \%)$, anticoagulants and heparin $(5.7 \%)$. Inadequate therapy monitoring (47.7\%), excessive dosage (28.5\%), drug-drug interactions (10.1\%) were the most common identified type of errors leading to preventable $A D E$.

Conclusions: $3.2 \%$ of admissions was caused by ADEs. $2.2 \%$ of hospitalized patients experienced ADEs. $62 \%$ of ADEs were potentially preventable. A high proportion of preventable ADEs were around a small number of drugs. Effective safety practices directed to reduce the incidence of medication errors are needed.

KEY WORDS: Medication errors. Adverse drug events. Preventability. Drug therapy/ adverse effects. Drug-related adverse reactions.

Sánchez Muñoz LA, Castiella Herrero J, Sanjuán Portugal FJ, Naya Manchado J, Alfaro Alfaro MJ. Utilidad del CMBD para la detección de acontecimientos adversos por medicamentos. An Med Interna (Madrid) 2007; 24: 113-119.

\section{INTRODUCCIÓN}

Los acontecimientos adversos medicamentosos (AAM) son una patología emergente que junto con el resto de efectos adversos originados por la atención sanitaria han pasado a ser una prioridad de las políticas de calidad de los sistemas de salud (1). El interés por la seguridad del paciente se incrementó tras la publicación en 1999 del informe del Institute of Medicine de EE.UU. "To err is human: building a safer health system". Este informe dio a conocer la importante

Trabajo aceptado: 15 de noviembre de 2006

Correspondencia: Luis Ángel Sánchez Muñoz. Unidad de Medicina Interna. Fundación Hospital Calahorra. Ctra. de Logroño, s/n. 26500 Calahorra. La Rioja. lsanchezmunoz@gmail.com 
repercusión de los errores asistenciales, incidiendo especialmente en los asociados al uso de fármacos por ser uno de los tipos de error más prevalente. Se estimó que los errores de medicación ocasionaron más de 7.000 muertes anuales, superando a las causadas por los accidentes de trabajo (2).

Un reflejo de la preocupación por la seguridad del paciente es que servicios sanitarios como el NHS británico elaborara en el año 2000 los informes "An organisation with a memory: report of an expert group on learning from adverse events in the NHS" (3) y "Building a safer NHS for patients" (4) y en el 2004 "Seven steps to patient safety" (5), que en EE.UU. en 2002 varias sociedades consensuaran el documento "Pathways for Medication Safety" (6), y que recientemente la Organización Mundial de la Salud (OMS) creara la "Alianza Mundial para la Seguridad del Paciente" (7). La seguridad de los pacientes es también una de las prioridades del actual Plan de Calidad del Ministerio de Sanidad y Consumo (8).

Los AAM motivan el 4,1 a 14,2\% de los ingresos hospitalarios (9-17). La incidencia estimada de AAM en pacientes hospitalizados se sitúa en torno al 7,5-10,9\% en dos estudios recientes $(18,19)$, aunque en la bibliografía oscila entre el 1,5 y el $35 \%$, debido a diferencias metodológicas entre los diferentes trabajos (20-22). Se estima que la incidencia de AAM precoces tras el alta hospitalaria es del 16\% (23). En nuestro país se han comunicado recientemente los resultados del Estudio Nacional sobre los Efectos Adversos ligados a la hospitalización (ENEAS), que se ha realizado en una muestra de 24 hospitales públicos españoles: aparecen efectos adversos en $8,4 \%$ de los pacientes hospitalizados, más de un tercio son AAM $(37,4 \%)$ y el $34,8 \%$ son evitables (24).

La OMS define como reacción adversa medicamentosa a todo efecto perjudicial y no deseado que se presenta después de la administración de un medicamento a las dosis normalmente utilizadas en el hombre para la profilaxis, diagnóstico o tratamiento de una enfermedad o con objeto de modificar su función biológica (25). En la actualidad, se prefiere ampliar esta definición utilizando la expresión Acontecimientos Adversos por Medicamentos (AAM), que incluyen cualquier daño grave o leve causado por el uso terapéutico de un medicamento de carácter prevenible (errores de medicación) o no prevenible (reacciones adversas a medicamentos) (26).

El impacto de los AAM en morbimortalidad y costes sanitarios y su potencial evitabilidad en el 19 a 70,4\% de los casos (9,27-33), hace necesario adoptar medidas preventivas que mejoren la seguridad de la utilización de los medicamentos. Para ello es necesario identificar previamente qué tipos de errores aparecen y en qué partes del proceso de utilización de medicamentos se producen (selección, prescripción, validación, dispensación, administración, seguimiento). En el ámbito hospitalario se utilizan distintos métodos complementarios para detectar AAM ya que, debido a la complejidad del sistema de utilización de los medicamentos, ninguno de ellos es capaz de detectar todos los AAM. Además de los métodos clásicos de detección (registro de intervenciones farmacéuticas, técnicas de observación, monitorización de señales de alerta, notificación voluntaria/fomentada) existen métodos indirectos, basados en la explotación de datos informatizados con los que se puede aumentar los índices de detección de AAM. La explotación del Conjunto Mínimo Básico de Datos (CMBD) permite la revisión retrospectiva de las historias clínicas con diagnósticos al alta de AAM, según los códigos de la Clasificación Internacional de Enfermedades (CIE-9-MC). El análisis sistemático del CMBD permite su empleo como indicador del impacto de la yatrogenia medicamentosa en el ámbito hospitalario. El objetivo de este trabajo es estimar la incidencia y características de los AAM notificados en el informe de alta hospitalaria, analizar su gravedad y los fármacos implicados, y evaluar su posible evitabilidad.

\section{MATERIAL Y MÉTODO}

Estudio retrospectivo basado en el análisis del CMBD de los pacientes dados de alta entre enero y diciembre de 2.005 en todos los servicios asistenciales de un hospital de ámbito comarcal. El número de pacientes hospitalizados durante ese período fue de 3.983. El estudio se centró en los pacientes en cuyo informe de alta se incluían los códigos asociados a efectos adversos por medicamentos según la Clasificación Internacional de Enfermedades (CIE-9-MC) (códigos E870-E876, E930-E949, E980-E989, 960-979). Se consideró AAM a cualquier daño grave o leve causado por el uso terapéutico (incluyendo la falta de uso) de un medicamento. Se consideraron AAM prevenibles los causados por errores de medicación, y AAM no prevenibles a los que se producen a pesar del uso apropiado de los medicamentos y que corresponden a las reacciones adversas por fármacos (RAM) (26).

Mediante la revisión de las historias clínicas se registraron: a) características del paciente; b) manifestaciones clínicas del AAM; c) medicación concomitante; d) lugar de detección; e) medicamentos implicados; f) severidad del AAM; g) evitabilidad; y h) tipificación de los AAM potencialmente prevenibles y no prevenibles.

Los AAM se clasificaron en extrahospitalarios cuando eran detectados en pacientes ambulatorios en el Servicio de Urgencias y motivaban su ingreso, e intrahospitalarios cuando aparecían durante la estancia hospitalaria. Las manifestaciones clínicas de los AAM fueron agrupadas según el diccionario de reacciones adversas de la OMS (34), y los fármacos implicados según la clasificación anatómica oficial de medicamentos (35). En el análisis de la evitabilidad de los AAM se utilizó la clasificación de Schumock y Thornton (36) modificada por Otero y cols (32) (Tabla I). Los AAM potencialmente evitables se consideraron errores de medicación y se clasificaron según los mismos autores en: falta de seguimiento, dosis elevada en medicamento con margen terapéutico estrecho, falta de tratamiento preventivo, dosis excesiva según las características del paciente, automedicación incorrecta e interacción medicamentosa (32). Los AAM inevitables o RAM se clasificaron en tipo A: predecibles y relacionados con el mecanismo de acción y dosis; y tipo B: no relacionados con el mecanismo de acción ni la dosis, de tipo inmunológico o idiosincrásico.

La severidad del AAM se graduó, según la clasificación del Sistema Español de Farmacovigilancia (37), en leve (no requiere medidas adicionales), moderada (requiere tratamiento o aumento de la monitorización), grave (pone en peligro la vida del paciente, causa ingreso en el hospital o lesión permanente) y fatal (contribuye directa o indirectamente a la muerte del paciente).

\section{RESULTADOS}

Se detectan AAM en un 4,01\% de las altas codificadas en el periodo de estudio $(n=160)$, obteniéndose un total de 175 AAM. En $45 \%$ de los episodios $(n=72)$ se trataba de AAM en el uso extrahospitalario de fármacos, que fueron detectados en Urgen- 
TABLA I

\section{ANÁLISIS DE LA EVITABILIDAD DE LOS AAM}

Clasificación de Schumock y cols. (36), modificada por Otero y cols. (32)

Un AAM se puede considerar prevenible cuando se pueden contestar afirmativa-

mente a una o más de las siguientes preguntas:

- ¿El medicamento implicado en el AAM es inapropiado considerando la situación clínica del paciente?

- ¿La dosis, la vía o la frecuencia de administración son inapropiadas considerando la edad, el peso o la patología subyacente del paciente?

- ¿La duración del tratamiento es inferior o superior a la establecida para la indicación que se trata?

- ¿Se ha omitido la realización de los controles clínicos necesarios para el seguimiento del tratamiento?

- ¿Había presentado previamente el paciente una reacción alérgica o un efecto similar con el medicamento o con otros con los que presente reactividad cruzada o que tengan el mismo mecanismo de acción?

- ¿El AAM se produjo como consecuencia de algún tipo de interacción?

- ¿Se determinaron concentraciones séricas del medicamento potencialmente tóxicas o valores anormales en otras pruebas de laboratorio utilizadas para controlar el tratamiento?

- ¿Se ha prescindido del tratamiento preventivo necesario para evitar la aparición del efecto adverso cumpliendo el paciente criterios para recibirlo?

- ¿El AAM se produjo por un cumplimiento errático de la prescripción?

- ¿Se ha producido el AAM por un error en la administración del medicamento?

- ¿El AAM deriva de una automedicación incorrecta?

- ¿Se ha producido el AAM por alguna otra circunstancia que pueda ser considerada un error?

cias o consultas externas y originaron un 3,22\% de los ingresos urgentes. En el 55\% de los episodios $(\mathrm{n}=88)$ el AAM se originaba durante la hospitalización, con una tasa de AAM intrahospitalarios del 2,20\%. Los episodios recuperados del CMBD procedían mayoritariamente de servicios médicos $(86,88 \%)$.

Los pacientes afectados por AAM (53,7\% mujeres y 46,3\% hombres), en un $81,87 \%$ eran mayores de 65 años (edad media de 72,3 años, rango 1-97 años) y tomaban una media de 5,9 fármacos (rango 1-16). Las características de los pacientes y el lugar de detección de los AAM se recogen en la tabla II.

Los fármacos más frecuentemente implicados en AAM se muestran en la tabla III.

\section{TABLA II}

\section{CARACTERÍSTICAS DE LOS PACIENTES CON AAM Y LUGAR} DE DETECCIÓN

\begin{tabular}{|c|c|c|c|}
\hline & $\begin{array}{c}\text { Total pacientes } \\
\text { con AAM } \\
(n=160)\end{array}$ & $\begin{array}{c}\text { Pacientes con AAM } \\
\text { ambulatorio } \\
(n=72 ; 45 \%)\end{array}$ & $\begin{array}{c}\text { Pacientes con AAM } \\
\text { durante hospitalización } \\
\quad(n=88 ; 55 \%)\end{array}$ \\
\hline \multicolumn{4}{|l|}{ Sexon $(\%)$} \\
\hline Hombres & $74(46,25 \%)$ & $32(43,24 \%)$ & $42(56,75 \%)$ \\
\hline Mujeres & $86(53,75 \%)$ & $40(46,51 \%)$ & $46(53,48 \%)$ \\
\hline \multicolumn{4}{|l|}{ Edad n (\%) } \\
\hline$<35$ años & $14(8,75 \%)$ & $6(42,85 \%)$ & $8(57,14 \%)$ \\
\hline $35-65$ & $15(9,37 \%)$ & $12(80,00 \%)$ & $3(20,00 \%)$ \\
\hline$>65$ años & $131(81,87 \%)$ & $54(41,22 \%)$ & $77(58,77 \%)$ \\
\hline
\end{tabular}

Valores expresados como $\mathrm{n}(\%)$.

AAM: acontecimientos adversos causados por medicamentos.
TABLA III

FÁRMACOS MÁS FRECUENTEMENTE IMPLICADOS EN AAM

\begin{tabular}{lccc}
\hline Grupo/fármaco & $\begin{array}{c}\text { AAM detectados } \\
\text { extrahospitalariamente }\end{array}$ & $\begin{array}{c}\text { AAM detectados en } \\
\text { la hospitalización }\end{array}$ & Total AAM \\
\hline Antiinfecciosos & $7(8,5 \%)$ & $35(37,6 \%)$ & $42(24,0 \%)$ \\
Clucocorticoides sistémicos & $1(1,2 \%)$ & $26(28,0 \%)$ & $27(15,4 \%)$ \\
AlNES y analgésicos & $17(20,7 \%)$ & $3(3,2 \%)$ & $20(11,4 \%)$ \\
Diureticos & $8(9,8 \%)$ & $10(10,8 \%)$ & $18(10,3 \%)$ \\
Digoxina & $12(14,6 \%)$ & $4(4,3 \%)$ & $16(9,1 \%)$ \\
Insulina + ADOS & $9(11,0 \%)$ & $1(1,1 \%)$ & $10(5,7 \%)$ \\
ACO + heparina & $8(9,8 \%)$ & $2(2,2 \%)$ & $10(5,7 \%)$ \\
IECA & $3(3,7 \%)$ & $3(3,2 \%)$ & $6(3,4 \%)$ \\
Betabloqueantes & $4(4,9 \%)$ & $2(2,2 \%)$ & $6(3,4 \%)$ \\
Otros* & $3(3,7 \%)$ & $2(2,2 \%)$ & $5(2,9 \%)$ \\
Opiáceos & $3(3,7 \%)$ & $1(1,1 \%)$ & $4(2,3 \%)$ \\
Calcioantagonistas & $2(2,4 \%)$ & $1(1,1 \%)$ & $3(1,7 \%)$ \\
Antidepresivos & $2(2,4 \%)$ & $0(0,0 \%)$ & $2(1,1 \%)$ \\
Benzodiacepinas & $2(2,4 \%)$ & $0(0,0 \%)$ & $2(1,1 \%)$ \\
Amiodarona & $1(1,2 \%)$ & $1(1,1 \%)$ & $2(1,1 \%)$ \\
Antiepilepticos & $0(0,0 \%)$ & $2(2,2 \%)$ & $2(1,1 \%)$ \\
Total & 82 & 93 & 175 \\
\hline
\end{tabular}

Resultados expresados en número (\%) de medicamentos implicados. En algún AAM estuvo implicado más de un medicamento, y algún medicamento originó más de un AAM.

AAM: acontecimientos adversos causados por medicamentos. ACO: anticoagulantes orales; ADO: antidiabéticos orales; AINE: antiinflamatorios no esteroideos; IECA: inhibidores de la enzima conversora de la angiotensina. Otros*: grupos o fármacos implicados con frecuencia < $3 \%$ en el total de AAM.

Los órganos o sistemas afectados con más frecuencia por los AAM se muestran en la figura 1. Los principales fármacos asociados a AAM y las manifestaciones clínicas originadas se muestra en la tabla IV.

El 38,1\% de los AAM fueron graves, 40,0\% moderados y $21,9 \%$ leves. Los AAM graves fueron detectados fundamentalmente en Urgencias (95\%), consistiendo en hemorragias digestivas por AINE, intoxicaciones digitálicas, insuficiencia renal prerrenal o diselectrolitemias por diuréticos, hipoglucemias por antidiabéticos o insulina y complicaciones hemorrágicas por sobredosificación de anticoagulantes. Los AAM leves y moderados predominaron en la hospitalización (Fig. 2).

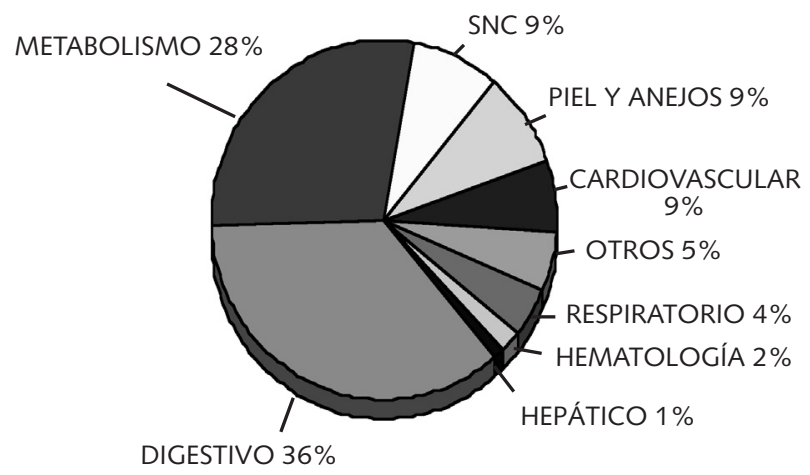

Fig. 1. Severidad de los episodios de AAM detectados. AAM: acontecimientos adversos causados por medicamentos. 


\section{TABLA IV}

\begin{tabular}{|c|c|c|}
\hline \multicolumn{3}{|c|}{$\begin{array}{l}\text { PRINCIPALES FÁRMACOS ASOCIADOS A AAM Y } \\
\text { MANIFESTACIONES CLIINICAS ORIGINADAS }\end{array}$} \\
\hline Fármacos & Manifestaciones clínicas & $n(\%)$ \\
\hline Antiinfecciosos & $\begin{array}{l}\text { Diarrea } \\
\text { Náuseas, vómitos } \\
\text { Rash cutáneo } \\
\text { Diarrea por C. difficile } \\
\text { Shock anafiláctico } \\
\text { Hepatotoxicidad } \\
\text { Candidiasis oral } \\
\text { Convulsión }\end{array}$ & $42(24,0 \%)$ \\
\hline Glucocorticoides sistémicos & $\begin{array}{l}\text { Hiperglucemia } \\
\text { Candidiasis }\end{array}$ & $27(15,4 \%)$ \\
\hline AINE + analgésicos & $\begin{array}{l}\text { Hemorragia digestiva alta } \\
\text { Urticaria/angioedema } \\
\text { Neutropenia } \\
\text { Úlcera gástrica } \\
\text { Anemia }\end{array}$ & $20(11,4 \%)$ \\
\hline Diuréticos & $\begin{array}{l}\text { Insuficiencia renal } \\
\text { Hipopotasemia } \\
\text { Hiponatremia } \\
\text { Hiperpotasemia } \\
\text { Acidosis metabólica } \\
\text { Crisis comicial } \\
\text { Somnolencia/síndrome confusional }\end{array}$ & $18(10,3 \%)$ \\
\hline Digoxina & $\begin{array}{l}\text { Hiporexia, náuseas, vómitos, } \\
\text { dolor abdominal } \\
\text { Síndrome confusional } \\
\text { Síncope } \\
\text { Bradiarritmia }\end{array}$ & $16(9,1 \%)$ \\
\hline Insulina + ADOs & $\begin{array}{l}\text { Hipoglucemia } \\
\text { Acidosis metabólica }\end{array}$ & $10(5,7 \%)$ \\
\hline ACO +heparina & $\begin{array}{l}\text { Hemorragia digestiva alta o baja } \\
\text { Hemoptisis } \\
\text { Hematoma abdominal } \\
\text { Púrpura }\end{array}$ & $10(5,7 \%)$ \\
\hline $\mathrm{IECA}$ & $\begin{array}{l}\text { Hiperpotasemia } \\
\text { Tos } \\
\text { Exantema cutáneo } \\
\text { Insuficiencia renal }\end{array}$ & $6(3,4 \%)$ \\
\hline Betabloqueantes & $\begin{array}{l}\text { Bradicardia, bradiarritmia, BAV, } \\
\text { hipotensión sintomáticas, o síncope } \\
\text { Broncoespasmo }\end{array}$ & $6(3,4 \%)$ \\
\hline Opiáceos & $\begin{array}{l}\text { Náuseas, íleo paralítico } \\
\text { Somnolencia/confusión } \\
\text { Insuficiencia respiratoria }\end{array}$ & $4(2,3 \%)$ \\
\hline Calcioantagonistas & $\begin{array}{l}\text { Rash cutáneo } \\
\text { BAV segundo grado }\end{array}$ & $3(1,7 \%)$ \\
\hline Antidepresivos & $\begin{array}{l}\text { Síncope } \\
\text { Somnolencia/confusión }\end{array}$ & $2(1,1 \%)$ \\
\hline
\end{tabular}

TABLA IV (CONT.)

\begin{tabular}{llc}
\multicolumn{3}{c}{ TABLA IV (CONT.) } \\
$\begin{array}{llc}\text { PRINCIPALES FÁRMACOS ASOCIADOS A AAM Y Y } \\
\text { MANIFESTACIONES CLÍNICAS ORIGINADAS }\end{array}$ \\
\hline Fármacos & Manifestaciones clínicas & $n(\%)$ \\
\hline Benzodiacepinas & Somnolencia/confusión & $2(1,1 \%)$ \\
\hline Amiodarona & Hipertiroidismo & $2(1,1 \%)$ \\
\hline Antiepiléptico & Vómitos & $2(1,1 \%)$ \\
& Somnolencia/confusión & \\
\hline Otros* & & $5(2,9 \%)$ \\
& & 175 \\
\hline
\end{tabular}

AAM: acontecimientos adversos causados por medicamentos. ACO: anticoagulantes orales; ADO: antidiabéticos orales; AINE: antiinflamatorios no esteroideos; IECA: inhibidores de la enzima conversora de la angiotensina. BAV: bloqueo aurículo-ventricular. Otros*: grupos o fármacos implicados con frecuencia $<3 \%$ en el total de AAM.

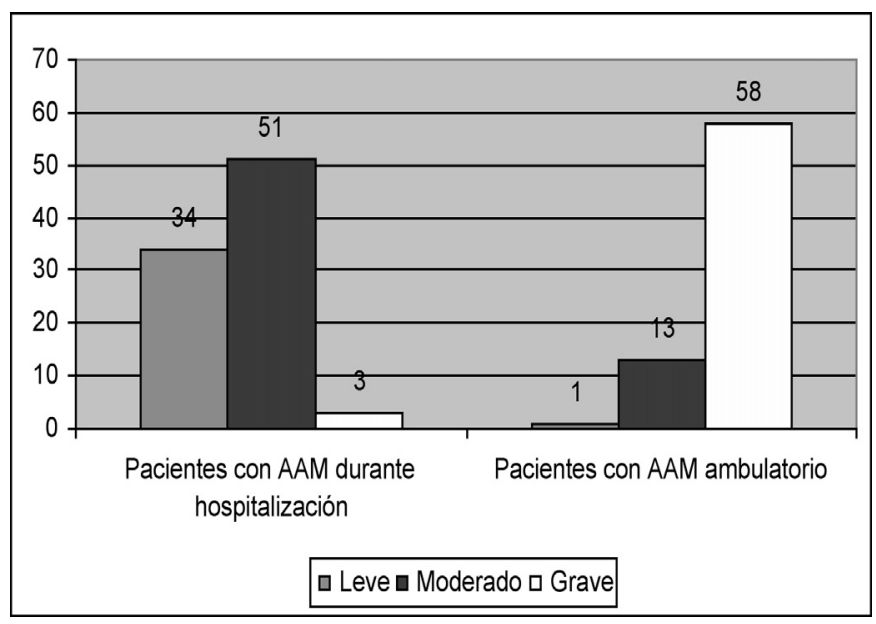

Fig. 2. Severidad de los episodios de AAM detectados. AAM: acontecimientos adversos causados por medicamentos.

El $62,3 \%$ de los AAM se consideraron prevenibles $(\mathrm{n}=$ 109) y por tanto, errores de medicación. De estos, $58,7 \%$ (n = 64) fueron AAM ocasionados por el consumo extrahospitalario de fármacos y $41,3 \%(\mathrm{n}=45)$ por el intrahospitalario. Por su frecuencia destacaron los ocasionados por glucocorticoides sistémicos (24,8\%), diureticos $(15,6 \%)$, digoxina $(14,7 \%)$, insulina y antidiabéticos orales $(9,2 \%)$, AINE y analgésicos $(9,2 \%)$, anticoagulantes orales y heparina $(8,3 \%)$, IECAS $(2,8 \%)$ y betabloqueantes $(2,8 \%)$. Las causas que motivaban su aparición fueron la falta de seguimiento $(47,7 \%)$, dosis elevada en medicamento con margen terapéutico estrecho $(14,7 \%)$, dosis excesiva según las características del paciente $(13,8 \%)$, interacción medicamentosa $(10,1 \%)$ y automedicación incorrecta $(6,4 \%)$.

El $37,7 \%$ de los AAM fueron RAM no prevenibles $(n=$ 66). En 71,2\% ( $=47)$ fueron RAM tipo A, relacionadas con el mecanismo de acción o la dosis del fármaco; en la mitad de los casos se trataba de diarrea secundaria a antibioterapia. Las 


\section{TABLA V}

CLASIFICACIÓN DE LOS AAM QUE SE CONSIDERARON PREVENIBLES

\begin{tabular}{lccc}
\hline & $\begin{array}{c}\text { AAM prevenibles } \\
\text { detectados en } \\
\text { Urgencias }\end{array}$ & $\begin{array}{c}\text { AAM prevenibles } \\
\text { durante la } \\
\text { hospitalización }\end{array}$ & $\begin{array}{c}\text { AAM prevenibles } \\
\text { totales }\end{array}$ \\
\hline $\begin{array}{l}\text { Falta de seguimiento } \\
\text { Dosis elevada en medicamento con } \\
\text { margen terapéutico estrecho }\end{array}$ & $14(21,9 \%)$ & $2(4,3 \%)$ & $16(14,7 \%)$ \\
Falta de tratamiento preventivo & $3(4,7 \%)$ & $0(0,0 \%)$ & $3(2,7 \%)$ \\
$\begin{array}{l}\text { Dosis excesiva según las características } \\
\text { del paciente }\end{array}$ & $6(9,4 \%)$ & $9(20,0 \%)$ & $15(13,8 \%)$ \\
Automedicación incorrecta & $7(10,9 \%)$ & $0(0,0 \%)$ & $7(6,4 \%)$ \\
Interacción medicamentosa. & $9(14,1 \%)$ & $2(4,4 \%)$ & $11(10,1 \%)$ \\
No indicado & $1(1,6 \%)$ & $0(0,0 \%)$ & $1(0,9 \%)$ \\
Accidental & $4(6,3 \%)$ & $0(0,0 \%)$ & $4(3,66 \%)$ \\
& 64 & 45 & $109)$ \\
\hline AAM: acon & & & \\
\end{tabular}

AAM: acontecimientos adversos causados por medicamentos.

RAM tipo $\mathrm{B}$, inmunológicas o idiosincráticas, fueron un $28,8 \%(n=19)$ y en su mayoria $(n=14)$ originadas por reacciones alérgico-anafilácticas de diferente gravedad a antiinfecciosos o analgésicos.

Los AAM originados en el uso extrahospitalario de fármacos y que motivaron el ingreso fueron 82 , de los cuales $64(78,04 \%)$ se consideraron evitables; fueron graves $80,6 \%$ de casos. Los AAM que acontecieron durante la hospitalización fueron 93, de los cuales $45(48,38 \%)$ se consideraron evitables; fueron graves $3,4 \%$ de casos. Los medicamentos implicados se muestran en la tabla III. Los errores cometidos en los AAM que se consideraron prevenibles se describen en la tabla V.

\section{DISCUSIÓN}

La importancia de los AAM queda reflejado en la creciente preocupación de profesionales y autoridades sanitarias ante la evidencia de que los AAM originan ingresos hospitalarios, prolongan la estancia hospitalaria, se asocian a un aumento de morbi-mortalidad, contribuyen al aumento de coste sanitario y son evitables en un importante porcentaje.

Resulta difícil comparar la incidencia de AAM entre los diversos estudios debido a que son diferentes las definiciones de AAM empleado, los subgrupos de población estudiados (ambulatorios, hospitalizados, tipo de hospital y servicio médico analizado) y los procedimientos de detección e intensidad de recogida de datos. Este es el motivo de la amplia variabilidad en las incidencias (1,5 a 35\%) recogidas en la bibliografía (20-22). La menor incidencia de AAM reflejada en nuestro trabajo $(4,01 \%$ total, $3,22 \%$ de los ingresos urgentes, $2,20 \%$ de los pacientes hospitalizados) respecto a la bibliografía, se debe a las limitaciones del propio método, por ser retrospectivo (con la subsiguiente pérdida de información), a su dependencia de la mención explícita del AAM en el informe médico de alta y que posteriormente sea correctamente codificado y, a las limitaciones de la clasificación CIE9 respecto de los términos relacionados con AAM (38). El análisis del CMBD es más útil para detectar los AAM que motivan el ingreso hospitalario, que los ocurridos durante la hospitalización, especialmente los relacionados con la prescripción y seguimiento.

Si bien esta infraestimación es un factor desfavorable, la explotación del CMBD presenta como gran ventaja el aportar de forma ágil (mensual) y mantenida una información básica sobre el impacto de la yatrogenia medicamentosa en el ámbito hospitalario, especialmente sobre AAM conocidos y prevenibles que se centran en un reducido número de fármacos $(39,40)$, mejorando la cifra de AAM encontrados en comparación con métodos de notificación voluntaria o búsquedas realizadas de forma manual (41). Además, dado que el CMBD proporciona una información homogénea a nivel nacional, también permite comparar incidencias entre centros y servicios del propio centro, para identificar posibles desproporciones en la frecuencia de determinados AAM, permitiendo priorizar acciones preventivas sobre los AAM con mayor morbimortalidad y que sean potencialmente evitables $(40,42-$ 45). Como contrapartida, los métodos de mayor rendimiento pueden aumentar la detección de AAM en más de diez veces (46), pero también exigen mayores medios, mayor consumo de tiempo, suelen estar limitados a períodos relativamente cortos y suelen necesitar personal y/o financiación expresa para su realización (38-40).

Nuestra serie coincide con la bibliografía en la mayor incidencia de AAM asociada al aumento de edad (81,87\% mayores de 65 años) y discretamente superior en mujeres $(53,7 \%)$. Los AAM son más prevalentes entre la población anciana, su pronóstico es más grave y origina mayor número de ingresos (47-51), con una mayor incidencia de AAM prevenibles (hasta el 50\%) (52,53). Las modificaciones farmacocinéticas y farmacodinámicas asociadas al envejecimiento, la mayor comorbilidad, el uso de varios fármacos durante períodos prolongados (media de 5,9 fármacos en nuestra serie, un paciente con 16 fármacos) y la frecuente presentación atípica del AAM condicionan un mayor riesgo de interacciones y de sobredosificación (54-56). Este problema va en aumento ya que el $16,6 \%$ de la población española es mayor de 65 años (INE, padrón municipal 2005) y se estima que en 2017 es probable que alcance el $18,95 \%$. Para explicar el mayor porcentaje de mujeres con AAM se han argumentado como posibles causas su mayor longevidad, un mayor consumo de medicamentos, sobredosificación con respecto al peso y una predisposición condicionada genéticamente, pero no se conoce en qué medida influye cada una (45).

Los fármacos implicados, como en otra series, coinciden con el tratamiento farmacológico de las enfermedades más prevalentes de nuestra sociedad: antibacterianos, glucocorticoides sistémicos, AINE y otros analgésicos, diureticos, digoxina, insulina y antidiabéticos orales, anticoagulantes orales y heparina, IECA y betabloqueantes (57). Los citostáticos no aparecen en nuestra serie debido a que nuestro centro no dispone de Servicio de Oncología.

Es destacable que en el Servicio de Urgencias se detectan el $95 \%$ de los AAM graves y que el 78,04\% de los AAM allí registrados son prevenibles $(48,38 \%$ en el caso de los intrahospitalarios). Estos valores coinciden con la tendencia reflejada en la literatura de que la prevenibilidad es menor en los estudios realizados en pacientes hospitalizados que en los estudios sobre AAM que motivan la atención en urgencias o el ingreso hospitalario (46). 
Las principales causas que motivaron la aparición de AAM fueron la falta de seguimiento, la dosis elevada en medicamento con margen terapéutico estrecho (en el caso de sobredosificación de digital, diuréticos, antidiabéticos), o según las características del paciente y la automedicación (en el caso de hemorragias digestivas por AINE). Son frecuentes los AAM motivados por falta de seguimiento destacando especialmente los detectados en el Servicio de Urgencias, por su frecuencia, gravedad y evitabilidad. La falta de educación sanitaria, las características de los pacientes (personas mayores, múltiples medicaciones), las deficiencias organizativas y la fragmentación del sistema asistencial público español, en el que un paciente recibe prescripciones por varios facultativos, favorece la polifarmacia, con un control de estos tratamientos mejorable tanto en el medio ambulatorio como en el hospitalario.

La información obtenida del análisis de los AAM debe emplearse para mejorar la seguridad en el uso de los medicamentos. Para ello se han propuesto como herramientas la creación de una cultura de seguridad no punitiva en las organizaciones sanitarias, la mejora de la organización, de los procesos y procedimientos, la formación continuada y el empleo de las nuevas tecnologías (prescripción electrónica, sistemas expertos, códigos de barras, dispensación automática) (46,58-60).

A la vista de los resultados de nuestro trabajo y de otros realizados en el ámbito nacional (14-17,22,24,32,38-
$40,45,46,48,51,57)$ es evidente la necesidad de que los facultativos conozcamos que muchos de los AAM prevenibles dependen de los procesos de prescripción y seguimiento de un número reducido de fármacos, lo cual nos debe ayudar a implantar barreras que disminuyan su frecuencia.

\section{CONCLUSIONES}

1. Una elevada proporción de AAM son prevenibles y se centran en un número reducido de fármacos; por ello es necesario implantar prácticas efectivas de seguridad para reducir los errores de medicación.

2. El análisis del CMBD es una herramienta útil para la monitorización periódica de los AAM y la posterior priorización de medidas preventivas. A pesar de sus limitaciones, facilita una información de características homogéneas entre centros y servicios sanitarios (CMBD) y permite monitorizar AAM de forma sistemática y con escaso consumo de recursos durante periodos prolongados.

\section{AGRADECIMIENTO}

A la Dra. Sofía Cuesta por su tiempo y ayuda en la explotación de la base de datos hospitalaria.

\section{Bibliografía}

1. Otero MJ, Domínguez A. Acontecimientos adversos por medicamentos: una patología emergente. Farm Hosp 2000; 24: 258-66.

2. Kohn LT, Corrigan JM, Donaldson MS, eds. To err is human: Building a safer health system. Committee on Health Care in America. Institute of Medicine. Washington (DC): National Academy Press, 1999.

3. Expert Group on Learning from Adverse Events in the NHS. An organisation with a memory: report of an expert group on learning from adverse events in the NHS. London: Stationery Office, 2000. (Accedido 20 septiembre 2006). Disponible en: www.doh.gov.uk/pdfs/org.pdf

4. Department of Health. Building a safer NHS for patients, 2001 (Accedido 20 septiembre 2006). Disponible en: http://www.dh.gov.uk/assetRoot/04/05/80/94/04058094.pdf. 2001.

5. National Patient Safety Agency. Seven steps to patient safety. 2004. (Accedido 20 septiembre 2006). Disponible en: http://www.npsa.nhs. uk/health/resources/7steps

6. American Hospital Association, Health Research \& Educational Trust, and the Institute for Safe Medication Practices. Pathways for Medication Safety.2002. (Accedido 20 septiembre 2006). Disponible en: http://www.ismp.org/Tools/PathwaySection1.pdf, http://www.ismp. org/Tools/PathwaySection2.pdf, http://www.ismp.org/Tools/PathwaySection3.pdf.

7. World Health Organization. World Alliance fo Patient Safety. Forward Programme 2005 (Accedido 20 septiembre 2006). Disponible en: http://www.who.int/patientsafety/en/brochure_final.pdf

8. Plan de Calidad para el Sistema Nacional de Salud . Marzo-06, Secretaría General de Sanidad. Agencia de Calidad del SNS. (Accedido 20 septiembre 2006). Disponible en: http://www.msc.es/novedades/docs/notaPrensa20060323TextoIntegro.pdf

9. Nelson KM, Talbert RL. Drug-related hospital admissions. Pharmacotherapy 1996;16:701-7.

10. Hallas J, Harvald B, Gram LF, Grodum E, Brosen K, Haghfelt T, et al. Drug related hospital admissions: the role of definitions and intensity of data collection, and the possibility of prevention. J Intern Med 1990; 228: 83-90.

11. Hallas J, Gram LF, Grodum E, Damsbo N, Brosen K, Haghfelt T, et al. Drug related admissions to medical wards: a population based survey. Br J Clin Pharmac 1992; 33: 61-8.
12. Dartnell JGA, Anderson RP, Chohan V, Galbraith KJ, Lyon MEH, Nestor PJ, et al. Hospitalisation for adverse events related to drug therapy: incidence, avoidability and costs. Med J Aust 1996; 164: 659-62.

13. Stanton LA, Peterson GM, Rumble RH, Cooper GM, Polack AE. Drug related admissions to an Australian hospital. J Clin Pharm Ther 1994; 19: 341-7.

14. Armadans L, Carné X, Laporte JR. Detección de reacciones adversas a medicamentos a partir del diagnóstico de ingreso hospitalario. Métodos y resultados. Med Clin (Barc) 1998; 91: 124-7.

15. Garijo B, De Abajo FJ, Castro MAS, Lopo CR, Carcas A, Frías J. Hospitalizaciones motivadas por fármacos: un estudio prospectivo. Rev Clin Esp 1991; 188: 7-12.

16. Ibáñez L, Laporte JR, Carné X. Adverse drug reactions leading to hospital admission. Drug Safety 1991; 6: 450-59.

17. Martín MT, Codina C, Tuset M, Carné X, Nogué S, Ribas J. Problemas relacionados con la medicación como causa del ingreso hospitalario. Med Clin (Barc) 2002; 118 (6): 205-10

18. Lazarou J, Pomeranz B, Corey P. Incidente of adverse drug reaction in hospitalizad patines. A metaanalisis of prospective studies. JAMA 1998; 279: 1200-5.

19. Baker GR, Norton PG, Flintoft V, Blais R, Brown A, Cox J, et al. The Canadian Adverse Events Study: the incidence of adverse events among hospital patients in Canada. CMAJ 2004; 170 (11): 1678-86

20. Cullen DJ, Sweitzer BJ, Bates DW, Burdick E, Edmondson A, Leape LL. Preventable adverse drug events in hospitalized patients: a comparative study of intensive care and general care units. Crit Care Med 1997; 25: 1289-97.

21. Nelson K, Talbert R. Drug related hospital admissions. Pharmacotherapy 1996; 16: 701-7.

22. Muñoz MJ, Ayani I, Rodríguez Sasiáin JM, Gutiérrez G, Aguirre C. Monitorización en un servicio de urgencias de reacciones adversas causadas por medicamentos en niños y adultos. Med Clin (Barc) 1998; 114: 92-8.

23. Forster AJ, Clark HD, Menard A, Dupuis N, Chernish R, Chandok N, et al. Adverse events among medical patients after discharge from hospital.. CMAJ 2004; 170 (3): 345-9 
24. Estudio Nacional sobre los Efectos Adversos ligados a la Hospitalización. ENEAS 2005. Informe Febrero 2006. Secretaría General de Sanidad. Dirección General de la Agencia de Calidad del Sistema Nacional de Salud. Ministerio de Sanidad y Consumo. Accesible en

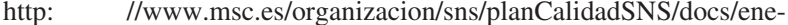
as2005Baja.pdf\#search=\%22ENEAS $\% 20$ filetype $\% 3$ Apdf $\% 22$

25. World Health Organization. Requirements for adverse reaction reporting. Geneva, Switzerland: World Health Organization, 1975.

26. Otero MJ, Codina C, Tamés MJ, Pérez M, et al. Errores de medicación: estandarización de la terminología y clasificación. Resultados de la Beca Ruiz-Jarabo 2000. Farm Hosp 2003; 27 (3): 137-149

27. Bates DW, Cullen DJ, Laird N, Petersen LA, Small SD, Servi D, et al. Incidence of adverse drug events and potencial adverse drug events. JAMA 1995; 274: 29-34.

28. Dennehy CE, Kishi DT, Louie C. Drug-related illness in emergency department patients. Am J Health-Syst Pharm 1996; 53: 1422-6.

29. Pearson TF, Pittman DG, Longley JM, Grapes T, Vigliotti DJ, Mullis SR. Factors associated with preventable adverse drug reactions. Am J Health- Syst Pharm 1994; 51: 2268-72.

30. Goettler M, Schneewiss S, Hasford J. Adverse drug reactions monitoring -cost and benefit considerations. Part II: cost and preventability of adverse drug reactions leading to hospital admission. Pharmacoepidemiol Drug Safety 1997; 6(Suppl 3): 79-90.

31. Seeger JD, Kong SX, Schumock GT. Characteristics associated with ability to prevent adverse drug reactions in hospitalized patients. Pharmacotherapy 1998; 18: 1284-9.

32. Otero MJ, Bajo A, Maderuelo JA, Domínguez-Gil A. Evitabilidad de los acontecimientos adversos inducidos por medicamentos detectados en un Servicio de Urgencias. Rev Clin Esp 1999; 199: 796-805.

33. Tafreshi MJ, Melby MJ, Kaback KR, Nord TC. Medication-related visits to the emergency department: a prospective study. Ann Pharmacother 1999; 33: 1252-7. related emergency department visits and hospital admissions. Am J Hosp Pharm 1992; 49: 1696-700.

34. WHO. Collaborating Centre for International Drug Monitoring. International monitoring of adverse drug reactions. Ginebra, 1988

35. Consejo General de Colegios Oficiales de Farmacéuticos. Catálogos de especialidades Farmacéuticas. Madrid, 1995.

36. Schumock GT, Thorton JP. Focusing on preventability of adverse drug reactions. Hosp Pharm 1992; 27: 538.

37. Anónimo. Centros Autónomos de Farmacovigilancia: Ministerio de Sanidad y Consumo, 1994.

38. Aguirre C. Utilización del CMBD hospitalario como fuente de información en farmacovigilancia. Libro resúmenes de las III Jornadas de Farmacovigilancia. Pág.30-35. (Accedido 20 septiembre 2006). Disponible en: http: //www.jccm.es/sanidad/reuniones/farmacovigilancia/libro.pdf

39. Rubio A, Perea S. Evaluación de reacciones adversas a medicamentos en un hospital general. Libro resúmenes de las III Jornadas de Farmacovigilancia. Pág.86. (Accedido 20 septiembre 2006). Disponible en: http: //www.jccm.es/sanidad/reuniones/farmacovigilancia/libro.pdf

40. Torelló J, Castillo JR, Mengíbar A, Vigil E. Análisis de las RAM registradas en el CMBD de los hospitales universitarios Virgen del Rocío (HHUUVR). Libro resúmenes de las III Jornadas de Farmacovigilancia. Pág.84. (Accedido 20 septiembre 2006). Disponible en: http: //www.jccm.es/sanidad/reuniones/farmacovigilancia/libro.pdf

41. Cullen DJ, Sweitzer BJ, Bates DW, Burdick E, Edmondson A, Leape LL. Preventable adverse drug events in hospitalized patients: a comparative study of intensive care and general care units. Crit Care Med 1997; 25: 1289-97.
42. Navarro-Calderón E, Navarro MJ, Muelas J, Escoms V, Rodríguez R, Salazar A. Importancia de la búsqueda activa en la detección de reacciones adversas medicamentosas. Seguim Farmacoter 2004; 2(1): 24 28.

43. Navarro-Calderón E, Navarro MJ, Muelas J, Salazar A. Exhaustividad de la declaración de reacciones adversas medicamentosas. Seguim Farmacoter 2004; 2(4): 244-249.

44. Navarro-Calderón E, Navarro MJ, Muelas J, Escoms V, Salazar A Utilidad del CMBD en la declaración de efectos adversos medicamentosos. Boletin epidemiológico semanal.Centro Nacional de Epidemiología. Semanas: 43-44 del 19/10 al 01/11 de 2003. 2003 vol. $11 \mathrm{n}^{\circ}$ 23/265-276

45. Corral S, Guerrero MD; Beltrán M, Salas J. Utilización del CMBD como herramienta para la detección de acontecimientos adversos a medicamentos. Farm Hosp (Madrid) 2004; 28 (4): 258-265

46. Otero MJ, Alonso P, Maderuelo JA, Garrido B, Domínguez A, Sánchez A. Acontecimientos adversos prevenibles causados por medicamentos en pacientes hospitalizados. Med Clin (Barc) 2006; 126 (3): 81-7.

47. Manesse C, Derkx FH, Ridder MA, Veld AJ, Van der Cammen TJ. Contribution of adverse drug reactions to hospital admisión of older patients. Age and Ageing 2000; 29: 35-39.

48. Sanchez M, Delgado L, Delgado E, Prieto S, Bermejo T. Detección y análisis de reacciones adversas a medicamentos en el servicio de Urgencias de un hospital general. Farm Hosp. 2006; 30 (2): 78-84.

49. Walker J, Wynne H. Review: The frequency and severity of adevrse drug reactions in elderly people. Age Aging. 1994; 23: 255-9

50. Roughead E, Gilbert A, Primrose J, Sansom L. Drug-related hospital admissions: a review of Australian studies published 1988-1996. Med J Aust 1998; 168: 405-8.

51. Vilà A, San José A, Roure C, Armadans L, Vilardell M, y Grupo para el estudio de las Reacciones Adversas a Medicamentos en pacientes mayores hospitalizados. Estudio multicéntrico prospectivo de reacciones adversas a medicamentos en pacientes ancianos hospitalizados. Med Clin (Barc) 2003; 120(16): 613-8

52. Thomas EJ, Brennan TA . Incidence and types of preventable adverse events in elderly patients: population based review of medical records. BMJ 2000; 320: 741-744.

53. Gurwitz JH, Field TS, Avorn J, McCormick D, Jain S, Benser M, et al. Incidence and preventability of adverse drug events in nursing homes. Am J Med 2000; 109: 87-94.

54. Seidl LG, Thornton GF, Smith JW, Cluff LE. Studies on the epidemiology of adverse drug reactions: reactions in patients in a general medical service. Bull John Hopkins Hosp 1966; 119: 299-315.

55. Carbonin P. Is age an independent risk factor of ADRs in hospitalised medical patients J Am Geriatr Soc 19991; 39: 1093-9.

56. Ajayi FO, Perry J. Adverse drug reaction: a review of relevant factors. J Clin Pharmacol 2000; 40: 1090-1101.

57. Puche E, Luna J. Reacciones adversas a medicamentos: una revisión actualizada del problema en España. Rev Clin Esp 2006; 206 (7): 336 9.

58. Oren E, Shaffer ER, Guglielmo BJ. Impact Of Emerging Technologies On Medication Errors And Adverse Drug Events. Am J Health-Syst Pharm 2003; 60: 1447- 58

59. Kuperman GJ, Gibson RF. Computer Physician Order Entry: Benefits, Costs, And Issues. Ann Intern Med 2003; 139: 31-39.

60. Kaushal R, Shojania Kg, Bates Dw. Efects Of Computer Physician Order Entry And Clinical Decision Support Systems On Medication Safety. A Systematic Review. Arch Intern Med 2003; 163: 1409-16. 